\title{
Cycle-Consistent Adversarial Autoencoders for Unsupervised Text Style Transfer
}

\author{
Yufang Huang ${ }^{1}$ Wentao Zhu ${ }^{2}$ Deyi Xiong ${ }^{3}$ Yiye Zhang $^{4}$ Changjian Hu$^{1}$ Feiyu Xu ${ }^{1}$ \\ ${ }^{1}$ Lenovo Research AI Lab, ${ }^{2}$ Kwai Inc., ${ }^{3}$ Tianjin University, ${ }^{4}$ Cornell University \\ \{yfhuang1992new,wentaozhu91\}@gmail.com, dyxiong@tju.edu.cn \\ yiz2014@med.cornell.edu, \{hucj1,fxu\}@lenovo.com
}

\begin{abstract}
Unsupervised text style transfer is full of challenges due to the lack of parallel data and difficulties in content preservation. In this paper, we propose a novel neural approach to unsupervised text style transfer which we refer to as Cycle-consistent Adversarial autoEncoders (CAE) trained from non-parallel data. CAE consists of three essential components: (1) LSTM autoencoders that encode a text in one style into its latent representation and decode an encoded representation into its original text or a transferred representation into a style-transferred text, (2) adversarial style transfer networks that use an adversarially trained generator to transform a latent representation in one style into a representation in another style, and (3) a cycle-consistent constraint that enhances the capacity of the adversarial style transfer networks in content preservation. The entire CAE with these three components can be trained end-to-end. Extensive experiments and in-depth analyses on two widely-used public datasets consistently validate the effectiveness of proposed CAE in both style transfer and content preservation against several strong baselines in terms of four automatic evaluation metrics and human evaluation.
\end{abstract}

\section{Introduction}

Unsupervised text style transfer is to rewrite a text in one style into a text in another style while the content of the text remains the same as much as possible without using any parallel data. Style transfer can be utilized in many tasks such as personalization in dialogue systems (Oraby et al., 2018; Colombo et al., 2019), sentiment and word decipherment (Shen et al., 2017), offensive language translation (Nogueira dos Santos et al., 2018), and data augmentation (Perez and Wang, 2017; Mikołajczyk and Grochowski, 2018; Zhu et al., 2020), etc.

However, there are a variety of challenges to text style transfer in practice. First, we do not have large-scale style-to-style parallel data to train a text style transfer model in a supervised way. Second, even with non-parallel corpora, the inherent discrete structure of text sequences aggravates the difficulty of learning desirable continuous representations for style transfer (Huang et al., 2020; Zhao et al., 2018; Bowman et al., 2016; Hjelm et al., 2018). Third, it is difficult to preserve the content of a text when its style is transferred. To obtain good content preservation for text style transfer, various disentanglement approaches (Shen et al., 2017; Hu et al., 2017; Fu et al., 2018; Sudhakar et al., 2019) are proposed to separate the content and style of a text in the latent space. However, content-style disentanglement is not easily achievable as content and style typically interact with each other in texts in subtle ways (Lample et al., 2019).

In order to solve the issues above, we propose a cycle-consistent adversarial autoencoders (CAE) for unsupervised text style transfer. In CAE, we learn the representation of a text where we embed both content and style in the same space. Such space is constructed for each style from non-parallel data. We then transfer the learned representation from one style space to another space. To guarantee that the content is preserved during the style transfer procedure, the transferred representation is transferred back to the original space to minimize the distance between its original representation and the reversely transferred representation.

This work is licensed under a Creative Commons Attribution 4.0 International License. License details: http:// 


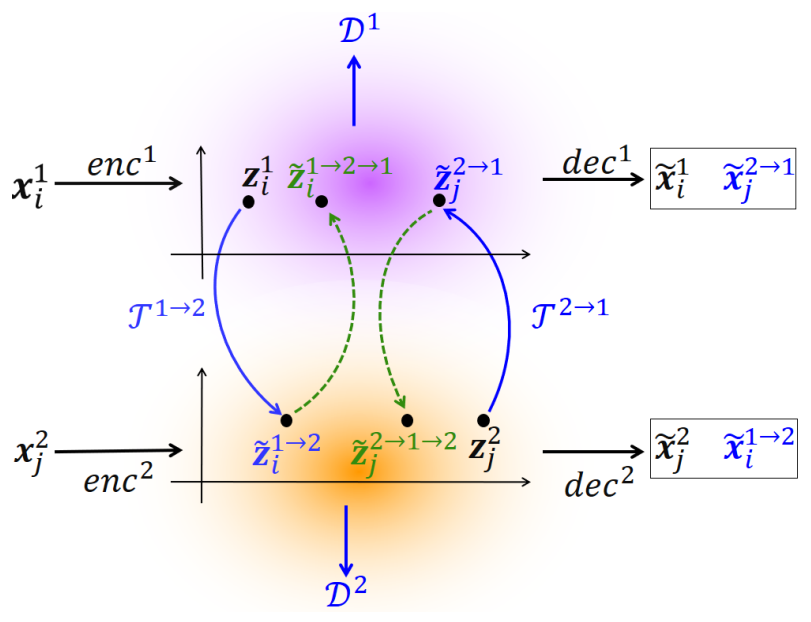

Figure 1: The architecture of the proposed CAE network.

Figure 1 demonstrates the diagram of the proposed CAE. Without loss of generality, we discuss CAE for text transfer between two styles. Multiple styles can be factorized into two styles (Shen et al., 2020a). Specifically, CAE is composed of three essential components: LSTM autoencoders, adversarial style transfer networks and a cycle-consistent constraint. The LSTM autoencoder contains an encoder enc to encode a sentence $\boldsymbol{x}_{i}^{s}$ from style $s$ into a hidden representation $\boldsymbol{z}_{i}^{s}$ in the corresponding style space and a decoder dec to generate sentences from vectors $\boldsymbol{z}_{i}^{s}$ learned by the LSTM encoder (or $\tilde{\boldsymbol{z}}_{j}$ transferred from the other style space). The adversarial style transfer networks learn a generator $\mathcal{T}$ to generate a representation $\tilde{\boldsymbol{z}}_{i}^{s_{1} \rightarrow s_{2}}$ in style space $s_{2}$ from $\boldsymbol{z}_{i}^{s_{1}}$ in style space $s_{1}$, or the other way around from style space $s_{2}$ to $s_{1}$. It also uses a discriminator to ensure that the transferred representations belong to the corresponding style space.

The top of Figure 2 displays the original sentences and style-transferred sentences generated by the LSTM decoder from transferred representations produced by the generator of the adversarial style transfer network. The cycle-consistent constraint transfers back representations to their original space and attempts to minimize their distances, as demonstrated in the bottom of Figure 2.

In summary, our contributions are threefold as follows.

- We propose a novel end-to-end framework with three components to learn text style transfer without using parallel data.

- To the best of our knowledge, our work is the first to use the cycle-consistent constraint in the latent representational space for unsupervised text style transfer.

- The proposed CAE are validated on two widely-used datasets: Yelp restaurant review sentiment transfer dataset and Yahoo QA topic transfer dataset. Extensive experiments and analyses demonstrate that CAE obtains better performance than several state-of-the-art baselines in both style transfer and content preservation.

\section{Related work}

A number of text style transfer approaches have been proposed in recent years following the pioneering study of style transfer in images (Gatys et al., 2015). These approaches can be roughly categorized into two strands: methods that disentangle representations of style and content and the others that do not.

In the first line of text style transfer, Hu et al. (2017) combine a variational autoencoder (VAE) with style discriminators to enforce that styles can be reliably inferred back from generated sentences. Shen et al. (2017) uses discriminators to align hidden states of the transferred samples from one style with the 


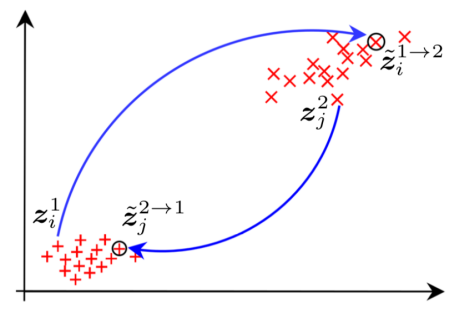

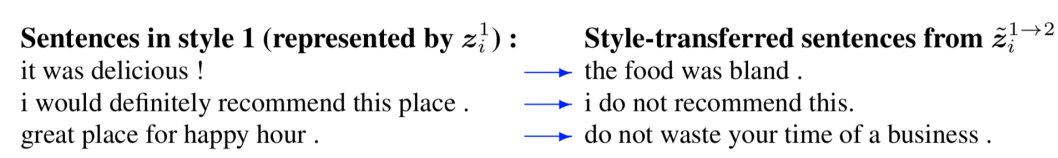

Sentences in style 2 (represented by $z_{j}^{2}$ ) : $\quad$ Style-transferred sentences from $\tilde{z}_{j}^{2 \rightarrow 1}$ : the prices are too high, the food is mediocre.$\longrightarrow$ the food was very attentive, excellent amazing . not going back to this place ever . $\longrightarrow$ definitely, this place a wonderful restaurant ! just returned from a disappointing meal . $\longrightarrow$ these food is awesome .

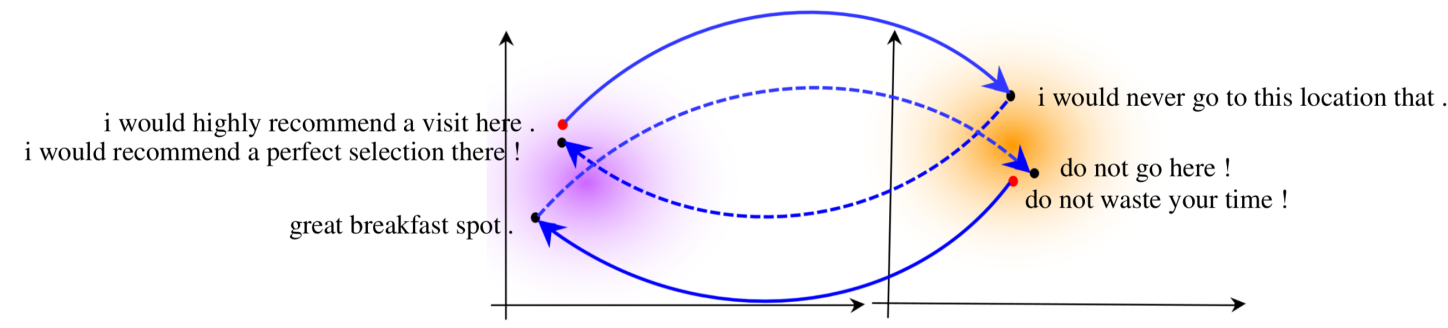

Figure 2: The visualization of style transfer and cycle-consistent constraint in CAE. Upper: sentences in one style and style-transferred sentences in another style. Bottom: cycle-consistent constraint enforcing that sentences transferred into a different style can be translated back to its original meaning in its original style.

true samples in the other to obtain the shared latent content distribution. Fu et al. (2018) use an adversarial network to separate content representations from style representations. Prabhumoye et al. (2018) fix the machine translation model and the encoder of the back-translation model to obtain content representations, then generate texts with classifier-guided style-specific generators. Li et al. (2018) extract content words by deleting style indicator words, then combine the content words with retrieved style words to construct the final output. Xu et al. (2018) use reinforcement learning to jointly train a neutralization module which removes style words based on a classifier and an emotionalization module. ARAE (Zhao et al., 2018) and DAAE (Shen et al., 2020b) train GAN-regularized latent representations to obtain styleindependent content representations, then decodes the content representations conditioned on style. $\mathrm{He}$ et al. (2020) presents a new probabilistic graphical model for unsupervised text style transfer.

In the second line of works that avoid disentangled representations of style and content, Lample et al. (2019) use back-translation technique on denoising autoencoder model with latent representation pooling to control the content preservation. Their experiments and analyses show that the contentstyle disentanglement is neither necessary nor always satisfied with practical requirements, even with the domain adversarial training that explicitly aims at learning disentangled representations. Style Transformer (Dai et al., 2019) uses Transformer as a basic module to train a style transfer system. DualRL (Luo et al., 2019) employs a dual reinforcement learning framework with two sequence-to-sequence models in two directions, using style classifier and back-transfer reconstruction probability as rewards.

We follow the second line and propose a novel method that makes no assumption on the latent representation disentanglement. But differently, we perform style transfer in the latent representational spaces of the source and target style. And inspired by CycleGAN (Zhu et al., 2017; Zhu et al., 2018) which uses a cycle loss on image style transfer to enforce the back-translation of a transferred image to be equivalent to the original image, we also impose a cycle-consistent constraint on our style transfer network. However, training style transfer networks with such a cycle constraint on discrete texts is quite different from those on images and non-trivial. In order to enable cycle training on texts, we project texts onto adversarially regularized latent space collectively learned by the LSTM autoencoders and adversarial transfer networks. Different from latent cross project with Euclidean distance for semi-supervised style transfer (Shang et al., 2019), we construct latent CycleGAN to generate high quality sentences for unsupervised style transfer. 


\section{CAE: Cycle-consistent Adversarial Autoencoders}

Suppose we have two non-parallel text datasets $\mathbb{X}^{1}=\left\{\boldsymbol{x}_{i}^{1}\right\}_{i=1}^{n}$ and $\mathbb{X}^{2}=\left\{\boldsymbol{x}_{j}^{2}\right\}_{j=1}^{m}$ with different styles $s_{1}$ and $s_{2}$. The CAE employs LSTM autoencoder models to encode discrete text sequences $\boldsymbol{x}_{i}^{1}, \boldsymbol{x}_{j}^{2}$ into representations $\boldsymbol{z}_{i}^{1}, \boldsymbol{z}_{j}^{2}$, and to generate sentences $\tilde{\boldsymbol{x}}_{i}^{1 \rightarrow 2}, \tilde{\boldsymbol{x}}_{j}^{2 \rightarrow 1}$ based on latent representations $\tilde{\boldsymbol{z}}_{i}^{1 \rightarrow 2}, \tilde{\boldsymbol{z}}_{j}^{2 \rightarrow 1}$ transferred by the adversarial transfer network $\mathcal{T}^{1 \rightarrow 2}, \mathcal{T}^{2 \rightarrow 1}$ from $\boldsymbol{z}_{i}^{1}, \boldsymbol{z}_{j}^{2}$.

\subsection{LSTM autoencoders}

We use an LSTM (Hochreiter and Schmidhuber, 1997) autoencoder to learn the latent representation of a text for each style. The encoder employs an LSTM recurrent neural network to map the input sequence to a latent representation with a fixed size, and the decoder utilizes the other LSTM network to generate an output sequence from a hidden representation (Sutskever et al., 2014). Given the $i$-th input text sequence $\boldsymbol{x}_{i}^{1}=\left(x_{i, 1}^{1}, x_{i, 2}^{1}, \cdots, x_{i, L}^{1}\right)$ in style $s_{1}$, the LSTM autoencoder for style $s_{1}$ can be formulated as:

$$
p\left(\tilde{\boldsymbol{x}}_{i}^{1} \mid \boldsymbol{x}_{i}^{1} ; e n c^{1}, \operatorname{dec}^{1}\right)=\prod_{t=1}^{L} p\left(\tilde{x}_{i, t}^{1} \mid \boldsymbol{z}_{i}^{1}, \tilde{\boldsymbol{x}}_{i,<t}^{1} ; e n c^{1}, \operatorname{dec}^{1}\right)
$$

where $\tilde{\boldsymbol{x}}_{i}^{1}=\left(\tilde{x}_{i, 1}^{1}, \cdots, \tilde{x}_{i, L}^{1}\right)$ is the reconstructed sequence with the same length $L$ as $\boldsymbol{x}_{i}^{1}, \boldsymbol{z}_{i}^{1}=e n c^{1}\left(\boldsymbol{x}_{i}^{1}\right)$ is the learned latent representation from the encoder $e n c^{1}, \tilde{\boldsymbol{x}}_{i,<t}^{1}$ are the tokens generated before $\tilde{x}_{i, t}^{1}$ and we start the decoder by a start-of-sentence symbol " $<$ bos $>$ " which is $\tilde{x}_{i,<1}^{1} \cdot p\left(\tilde{x}_{i, t}^{1} \mid \boldsymbol{z}_{i}^{1}, \tilde{\boldsymbol{x}}_{i,<t}^{1} ;\right.$ enc $^{1}$, dec $\left.^{1}\right)$ is the softmax output from decoder $d e c^{1}$. For style $s_{2}$, similarly, we construct the other LSTM autoencoder with encoder $e n c^{2}$ and decoder $d e c^{2}$ to learn latent representation $\boldsymbol{z}_{j}^{2}$.

The LSTM autoencoder tries to reconstruct the input sequence $\boldsymbol{x}_{i}^{k}$ with the output $\tilde{\boldsymbol{x}}_{i}^{k}$ from the networks $e n c^{k}, d e c^{k}$, where $k=1,2$ for different styles. The training objective function for the two LSTM autoencoders can be computed as:

$\mathcal{L}_{R}\left(e n c^{1}, \operatorname{dec}^{1}, e n c^{2}, \operatorname{dec}^{2}\right)=-\frac{1}{n} \sum_{i=1}^{n} \log p\left(\tilde{\boldsymbol{x}}_{i}^{1}=\boldsymbol{x}_{i}^{1} \mid \boldsymbol{x}_{i}^{1} ; e n c^{1}, \operatorname{dec}^{1}\right)-\frac{1}{m} \sum_{j=1}^{m} \log p\left(\tilde{\boldsymbol{x}}_{j}^{2}=\boldsymbol{x}_{j}^{2} \mid \boldsymbol{x}_{j}^{2} ; e n c^{2}, \operatorname{dec}^{2}\right)$

The two LSTM autoencoders transform discrete sequences into latent continuous representations, which facilitate the style transfer models to perform style transfer and cycle training in the continuous space.

\subsection{Adversarial style transfer networks}

Once we obtain the representations of text sequences in different styles via LSTM autoencoders, we learn two transformation functions $\mathcal{T}^{1 \rightarrow 2}$ and $\mathcal{T}^{2 \rightarrow 1}$ to map a representation in one style to the representation in the other style in the learned latent spaces. The style transfer in this way is formulated as:

$$
\tilde{\boldsymbol{z}}^{1 \rightarrow 2}=\mathcal{T}^{1 \rightarrow 2}\left(\boldsymbol{z}^{1}\right), \quad \tilde{\boldsymbol{z}}^{2 \rightarrow 1}=\mathcal{T}^{2 \rightarrow 1}\left(\boldsymbol{z}^{2}\right)
$$

where $\tilde{\boldsymbol{z}}^{1 \rightarrow 2}$ is the generated latent representation in style $s_{2}$ from its original representation $z^{1}$ in style $s_{1}$ by the transformation $\mathcal{T}^{1 \rightarrow 2}$, and $\tilde{\boldsymbol{z}}^{2 \rightarrow 1}$ is the generated latent representation in style $s_{1}$ by $\mathcal{T}^{2 \rightarrow 1}$.

We use generative adversarial networks (Goodfellow et al., 2014) to learn the two transformation functions. Let's consider the learning of the transformation $\mathcal{T}^{1 \rightarrow 2}$. We regard the function $\mathcal{T}^{1 \rightarrow 2}$ as the generator that generates a representation in style $s_{2}$ from a representation in style $s_{1}$. We then build a discriminator $\mathcal{D}^{2}$ to distinguish representations in style $s_{2}$ from others. The generator tries to generate a representation that is able to fool the discriminator. The adversarial learning of the generator $\mathcal{T}^{1 \rightarrow 2}$ and the discriminator $\mathcal{D}^{2}$ is formulated as:

$$
\begin{aligned}
\min _{\mathcal{T}^{1 \rightarrow 2}} \max _{\mathcal{D}^{2}} \mathcal{L}_{G}\left(\mathcal{T}^{1 \rightarrow 2}, \mathcal{D}^{2}\right) & =\mathbb{E}_{\boldsymbol{z}^{2} \sim p_{\boldsymbol{z}^{2}}}\left[\log \mathcal{D}^{2}\left(\boldsymbol{z}^{2}\right)\right]+\mathbb{E}_{\boldsymbol{z}^{1} \sim p_{\boldsymbol{z}^{1}}}\left[\log \left(1-\mathcal{D}^{2}\left(\mathcal{T}^{1 \rightarrow 2}\left(\boldsymbol{z}^{1}\right)\right)\right)\right] \\
= & \mathbb{E}_{\boldsymbol{x}^{2} \sim p_{\text {data }}}\left[\log \mathcal{D}^{2}\left(e n c^{2}\left(\boldsymbol{x}^{2}\right)\right)\right]+\mathbb{E}_{\boldsymbol{x}^{1} \sim p_{\text {data }}}\left[\log \left(1-\mathcal{D}^{2}\left(\mathcal{T}^{1 \rightarrow 2}\left(\operatorname{enc}^{1}\left(\boldsymbol{x}^{1}\right)\right)\right)\right)\right]
\end{aligned}
$$

Similarly, we can derive the generative adversarial loss $\mathcal{L}_{G}\left(\mathcal{T}^{2 \rightarrow 1}, \mathcal{D}^{1}\right)$ for style transformation function $\mathcal{T}^{2 \rightarrow 1}$ and discriminator $\mathcal{D}^{1}$. 


\subsection{Cycle-consistent constraint}

Theoretically, the adversarial style transfer networks described above are capable of learning many different transformation functions that can generate outputs in the distribution identical to the target style space (Zhu et al., 2017). This is because that the learning of the transformation functions lacks of sufficient constraints and the two functions are learned in a relatively separate way according to equations (4) and (5).

In order to learn desirable transformation functions, we use a cycle-consistent constraint to tighten the learning of the two transformation functions $\mathcal{T}^{1 \rightarrow 2}$ and $\mathcal{T}^{2 \rightarrow 1}$, which is inspired by CycleGAN (Zhu et al., 2017). The cycle-consistent constraint expects that a transferred representation generated by a transformation function can be translated back to its original representation by the other transformation function.

Given a latent representation $\boldsymbol{z}^{1}$ in style $s_{1}$, the reconstructed latent representation through the two style transformation functions $\mathcal{T}^{1 \rightarrow 2}, \mathcal{T}^{2 \rightarrow 1}$ can be obtained as:

$$
\tilde{\boldsymbol{z}}^{(1 \rightarrow 2) \rightarrow 1}=\mathcal{T}^{2 \rightarrow 1}\left(\tilde{\boldsymbol{z}}^{1 \rightarrow 2}\right)=\mathcal{T}^{2 \rightarrow 1}\left(\mathcal{T}^{1 \rightarrow 2}\left(\boldsymbol{z}^{1}\right)\right)
$$

Similarly, we can obtain the reconstructed latent representation $\tilde{\boldsymbol{z}}^{(2 \rightarrow 1) \rightarrow 2}$ for latent representation $\boldsymbol{z}^{2}$ in style $s_{2}$.

To constrain the transformation functions $\mathcal{T}^{2 \rightarrow 1}$ and $\mathcal{T}^{1 \rightarrow 2}$, the latent representational cycle-consistent reconstruction loss can be formulated as:

$$
\begin{aligned}
\mathcal{L}_{C}\left(\mathcal{T}^{2 \rightarrow 1}, \mathcal{T}^{1 \rightarrow 2}\right)= & \mathbb{E}_{\boldsymbol{z}^{1} \sim p_{\boldsymbol{z}^{1}}}\left[\left\|\tilde{\boldsymbol{z}}^{(1 \rightarrow 2) \rightarrow 1}-\boldsymbol{z}^{1}\right\|_{1}\right]+\mathbb{E}_{\boldsymbol{z}^{2} \sim p_{\boldsymbol{z}^{2}}}\left[\left\|\tilde{\boldsymbol{z}}^{(2 \rightarrow 1) \rightarrow 2}-\boldsymbol{z}^{2}\right\|_{1}\right] \\
= & \mathbb{E}_{\boldsymbol{x}^{1} \sim p_{\text {data }}}\left[\left\|\mathcal{T}^{2 \rightarrow 1}\left(\mathcal{T}^{1 \rightarrow 2}\left(\operatorname{enc}^{1}\left(\boldsymbol{x}^{1}\right)\right)\right)-\operatorname{enc}{ }^{1}\left(\boldsymbol{x}^{1}\right)\right\|_{1}\right] \\
& +\mathbb{E}_{\boldsymbol{x}^{2} \sim p_{\text {data }}}\left[\left\|\mathcal{T}^{1 \rightarrow 2}\left(\mathcal{T}^{2 \rightarrow 1}\left(\operatorname{enc}^{2}\left(\boldsymbol{x}^{2}\right)\right)\right)-\operatorname{enc} c^{2}\left(\boldsymbol{x}^{2}\right)\right\|_{1}\right]
\end{aligned}
$$

where $\|\cdot\|_{1}$ is $L_{1}$ norm.

This latent representational cycle-consistent reconstruction imposes the constraint on the adversarial style transfer networks to palliate mode-dropping in the latent style transfer, and to improve the content preservation in the generated sentences.

\subsection{Training and inference}

As CAE has three components in its network architecture, the end-to-end training objective of CAE is composed of three sub-objectives and is formulated as:

$$
\mathcal{L}_{C A E}=\lambda_{1} \mathcal{L}_{R}\left(e n c^{1}, \operatorname{dec}^{1}, e n c^{2}, \operatorname{dec} c^{2}\right)+\lambda_{2}\left(\mathcal{L}_{G}\left(\mathcal{T}^{2 \rightarrow 1}, \mathcal{D}^{1}\right)+\mathcal{L}_{G}\left(\mathcal{T}^{1 \rightarrow 2}, \mathcal{D}^{2}\right)\right)+\lambda_{3} \mathcal{L}_{C}\left(\mathcal{T}^{2 \rightarrow 1}, \mathcal{T}^{1 \rightarrow 2}\right)
$$

where $\lambda_{1}, \lambda_{2}$ and $\lambda_{3}$ control the relative importance of the three sub-objectives. We aim to solve:

$$
e n c^{1}, \operatorname{dec} c^{1}, e n c^{2}, \operatorname{dec} c^{2}, \mathcal{T}^{2 \rightarrow 1}, \mathcal{T}^{1 \rightarrow 2}, \mathcal{D}^{1}, \mathcal{D}^{2}=\arg \min _{\left\{e n c^{1}, d e c^{1}, e n c^{2}, d e c^{2}, \mathcal{T}^{2 \rightarrow 1}, \mathcal{T}^{1 \rightarrow 2}\right\}} \max _{\left\{\mathcal{D}^{1}, \mathcal{D}^{2}\right\}} \mathcal{L}_{C A E}
$$

For the inference, let's consider the transfer of a text $\boldsymbol{x}_{i}^{1}$ in style $s_{1}$ into a text in style $s_{2}$. We first obtain latent representation $\boldsymbol{z}_{i}^{1}=e n c^{1}\left(\boldsymbol{x}_{i}^{1}\right)$ using encoder $e n c^{1}$. We then perform style transfer and obtain the transferred latent representation $\tilde{\boldsymbol{z}}_{i}^{1 \rightarrow 2}$ in style $s_{2}$ based on equation (3). Finally, we employ the decoder $d e c^{2}$ to generate a transferred sequence $\tilde{\boldsymbol{x}}_{i}^{1 \rightarrow 2} \sim \operatorname{dec} c^{2}\left(\tilde{\boldsymbol{z}}_{i}^{1 \rightarrow 2}\right)$ in style $s_{2}$ :

$$
\tilde{x}_{i, t}^{1 \rightarrow 2}=\underset{\tilde{x}_{i, t}^{1 \rightarrow 2}}{\arg \max } p\left(\tilde{x}_{i, t}^{1 \rightarrow 2} \mid \tilde{\boldsymbol{z}}^{1 \rightarrow 2}, \tilde{\boldsymbol{x}}_{i,<t}^{1 \rightarrow 2} ; d e c^{2}\right)
$$

where $\tilde{\boldsymbol{x}}_{i}^{1 \rightarrow 2}=\left(\tilde{x}_{i, 1}^{1 \rightarrow 2}, \cdots, \tilde{x}_{i, L^{\prime}}^{1 \rightarrow}\right)$ with length $L^{\prime}, p\left(\cdot \mid \boldsymbol{z}, \cdot ; d e c^{2}\right)$ is the same as equation (1) calculated by the softmax from $d e c^{2}$ with previous tokens. The inference of the entire sequence $\tilde{\boldsymbol{x}}_{i}^{1 \rightarrow 2}$ in style $s_{2}$ from sequence $\boldsymbol{x}_{i}^{1}$ in style $s_{1}$ is formulated as:

$$
\tilde{\boldsymbol{x}}_{i}^{1 \rightarrow 2} \sim \operatorname{dec}^{2}\left(\tilde{\boldsymbol{z}}_{i}^{1 \rightarrow 2}\right)=\operatorname{dec}^{2}\left(\mathcal{T}^{1 \rightarrow 2}\left(\boldsymbol{z}_{i}^{1}\right)\right)=\operatorname{dec}^{2}\left(\mathcal{T}^{1 \rightarrow 2}\left(\operatorname{enc}{ }^{1}\left(\boldsymbol{x}_{i}^{1}\right)\right)\right)
$$

Similarly, we can conduct style transfer from a sequence $\boldsymbol{x}_{j}^{2}$ in style $s_{2}$ to generate a sequence $\tilde{\boldsymbol{x}}_{j}^{2 \rightarrow 1}$ in style $s_{1}$. 


\begin{tabular}{c|c|c|c|c}
\hline Dataset & \multicolumn{2}{|c|}{ Yelp } & \multicolumn{2}{c}{ Yahoo } \\
\hline Styles & Positive & Negative & Entertainment \& Music & Politics \& Government \\
\hline \#Sent. & $382 \mathrm{~K}$ & $252 \mathrm{~K}$ & $441 \mathrm{~K}$ & $153 \mathrm{~K}$ \\
\hline \#Vocab. & \multicolumn{2}{|c|}{$10 \mathrm{~K}$} & \multicolumn{2}{c}{$116 \mathrm{~K}$} \\
\hline \#Pruned Vocab. & \multicolumn{2}{|c|}{$10 \mathrm{~K}$} & \multicolumn{2}{c}{$30 \mathrm{~K}$} \\
\hline
\end{tabular}

Table 1: Statistics of Yelp review sentiment transfer dataset and Yahoo QA topic transfer dataset.

\section{Experiments}

To compare our work with previous approaches to text style transfer from non-parallel data, we conducted experiments on two text transfer tasks: sentiment transfer on the Yelp restaurant review corpus and topic transfer on the "Yahoo! Answers Comprehensive Questions and Answers version 1.0" dataset. We also carried out ablation experiments to study the impact of different components of CAE on overall performance of style transfer.

\subsection{Experimental setup}

\subsubsection{Datasets}

For the Yelp dataset, we followed the same experimental setup and used the same dataset as Crossaligned auto-encoder (Shen et al., 2017) and ARAE (Zhao et al., 2018) for sentiment transfer on the Yelp restaurant reviews. The sentiment of a review is labeled as positive if the rating is above three; otherwise, it is labeled as negative. We used $70 \%$ of the data for training, $10 \%$ for validation and the rest for testing.

For the Yahoo QA dataset, we chose two topics for style transfer: "Entertainment \& Music" and "Politics \& Government", and extracted questions from these two topics to construct the final dataset. The partition ratios of this dataset for training and testing are $80 \%$ and $20 \%$, respectively. To reduce the vocabulary size, we pruned the vocabulary to keep the most frequent words and replaced other words with " $<$ unk $>$ ". Table 1 shows the statistics of the two datasets.

\subsubsection{Baselines}

We compared CAE with the following baselines: (1) LSTM autoencoder (AE): using only LSTM autoencoders in CAE for the two styles without the style transfer networks and cycle-consistent constraint. (2) Cross-aligned autoencoder (Cross-aligned AE) (Shen et al., 2017): aligning the hidden states of autoencoders adversarially to learn a shared latent content distribution. (3) ARAE (Zhao et al., 2018): adversarially training GAN-regularized prior with a classifier to obtain style-independent content representations, then conducting style transfer through decoders conditioned on style. (4) Template-based method (Li et al., 2018): replacing the style words of source sentence with the other style words retrieved from target sentences. (5) Cycled reinforcement learning approach (Cycled RL) (Xu et al., 2018): using reinforcement learning to jointly train a neutralization module which removes style words based on a classifier and an emotionalization module.

\subsubsection{Hyper-parameter settings}

The used encoders $e n c^{1}, e n c^{2}$ and decoders $d e c^{1}, d e c^{2}$ were LSTM networks with one hidden layer of size $h_{n}=128$ on the Yelp review dataset and of size $h_{n}=300$ on Yahoo QA dataset. The word embedding size was the same as the number of hidden neurons $h_{n}$. The latent variables $\boldsymbol{z}^{1}, \boldsymbol{z}^{2}, \tilde{\boldsymbol{z}}^{2 \rightarrow 1}$ and $\tilde{\boldsymbol{z}}^{1 \rightarrow 2}$ were $L_{2}$-normalized to 1 . The transformation functions $\mathcal{T}^{1 \rightarrow 2}, \mathcal{T}^{2 \rightarrow 1}$ were parameterized by two-layer fully-connected neural networks $\left(h_{n}-h_{n}-h_{n}\right.$ neurons). The discriminators $\mathcal{D}^{1}, \mathcal{D}^{2}$ were twolayer fully-connected neural networks ( $h_{n}-h_{n}-1$ neurons) with hyperbolic tangent activation function in the first layer and sigmoid activation function for the second layer. The $\lambda_{1}, \lambda_{2}, \lambda_{3}$ were set as $0.1,1.0$, 1.0 respectively based on the performance on the validation set.

\subsubsection{Evaluation metrics}

We used four automatic metrics to quantitatively evaluate the proposed CAE: Transfer, BLEU, PPL and RPPL, which have been widely used in previous literature (Zhao et al., 2018). Transfer is the style 


\begin{tabular}{c|cccc|cccc}
\hline Dataset & \multicolumn{4}{|c|}{ Yelp } & \multicolumn{4}{c}{ Yahoo } \\
\hline Models & Transfer $\uparrow$ & BLEU $\uparrow$ & PPL $\downarrow$ & RPPL $\downarrow$ & Transfer & BLEU & PPL & RPPL \\
\hline AE & $59.3 \%$ & 37.28 & 31.9 & 68.9 & $62.0 \%$ & 32.2 & 62.7 & 57.3 \\
\hline Template & $80.2 \%$ & $\mathbf{5 2 . 9 0}$ & 161.1 & 64.0 & $\mathbf{8 5 . 3 \%}$ & $\mathbf{5 8 . 9}$ & 137.5 & 178.4 \\
\hline Cycled RL & $81.4 \%$ & 22.00 & 81.9 & 85.6 & $75.0 \%$ & 20.2 & 37.5 & 305.7 \\
\hline Cross-aligned AE & $77.1 \%$ & 17.75 & 65.9 & 124.2 & $73.4 \%$ & 11.5 & 36.5 & 79.3 \\
\hline ARAE & $81.8 \%$ & 20.18 & 27.7 & 77.0 & $77.7 \%$ & 21.1 & 34.4 & 53.7 \\
\hline CAE & $\mathbf{8 6 . 9 \%}$ & 22.51 & $\mathbf{2 1 . 6}$ & $\mathbf{5 7 . 0}$ & $83.8 \%$ & 23.0 & $\mathbf{2 5 . 4}$ & $\mathbf{5 2 . 6}$ \\
\hline
\end{tabular}

Table 2: Quantitative comparisons. Left: results on Yelp restaurant review sentiment transfer dataset, right: result on Yahoo QA topic transfer dataset.

transfer success rate and implemented as a classifier which is trained by the fastText library (Joulin et al., 2017). BLEU is used to evaluate the content preservation between the source sequence and transferred sequence (Papineni et al., 2002). To evaluate the fluency of the transferred sequence, we utilized the perplexity of the generated text denoted by PPL. We also used the reverse perplexity (RPPL) to assess the representativeness of generated texts with respect to the underlying data distribution and to detect the mode collapse for generative models (Zhao et al., 2018). RPPL scores were calculated by training an RNN language model on generated samples to evaluate the perplexity on real-world hold-out data (Zhao et al., 2018). We used the code from Zhao et al. (Zhao et al., 2018) (word embedding size of 300 with dropout 0.2 , and one-layer LSTM of size 300 with dropout 0.2 ) to build the language models and calculate PPL and RPPL. These four evaluation metrics together form a comprehensive evaluation and comparison between different approaches.

We also conducted human evaluation. We randomly chose 200 instances from each style for the human evaluation. Four human annotators can proficiently understand English texts and have sufficient background knowledge about this evaluation task. The annotation is blind to them in random order. They grade all sentences with scores from one to five for style transfer, content preservation and fluency. Following Wu et al. (2019) and Li et al. (2018), we regard a style transfer with scores larger than or equal to four on all three measures (style transfer, content preservation and fluency) as a successful transfer. We calculate the percentage of successful transfers and refer to this percentage as Suc.

\subsection{Results}

\subsubsection{Yelp restaurant reviews sentiment transfer}

The results are shown in Table 2 (left), from which we clearly observe that CAE obtains better performance than the five baseline approaches. Specifically, CAE yields improvements of 5.1, 6.1 and 7.0 points over the best baselines for sentiment transfer in terms of transfer success rate (Transfer), fluency (PPL) and mode reservation (RPPL), respectively.

The template-based method achieves the highest BLEU score since all content words are guaranteed to be kept by templates with only style words replaced by retrieved words. However, it obtains the worst perplexity, indicating that it is very difficult for the template-based method to generate fluent sentences. By contrast, our CAE achieves the lowest perplexity due to the strong LSTM decoders. The cycleconsistent constraint enables CAE to yield the best RPPL as it palliates mode dropping in style transfer. Additionally, the adversarial style transfer network constrained by the cycle consistency loss facilitates CAE to perform well on both style transfer and content preservation.

\subsubsection{Yahoo questions topic transfer}

We further evaluated CAE against five basedlines on the Yahoo QA topic transfer task. Results in Table 2 (right) show that CAE obtains better Transfer $(+6.1 \%)$, PPL $(-9)$, BLEU and RPPL than the best baseline approaches of Cycled RL, Cross-aligned AE and ARAE, demonstrating the advantages of CAE on style transfer, fluency and content preservation. 


\begin{tabular}{c||ccc|c||ccc|c}
\hline \multicolumn{1}{c||}{ Dataset } & \multicolumn{4}{c||}{ Yelp } & \multicolumn{4}{c}{ Yahoo } \\
\hline Models & Style $\uparrow$ & Content $\uparrow$ & Fluency $\uparrow$ & Suc $\uparrow$ & Style & Content & Fluency & Suc \\
\hline Cross-aligned AE & 4.09 & 3.99 & 4.14 & $52 \%$ & 3.90 & 3.90 & 3.85 & $40 \%$ \\
ARAE & 4.03 & 4.15 & 4.36 & $55 \%$ & 3.99 & 4.10 & 4.25 & $51 \%$ \\
CAE & $\mathbf{4 . 1 9}$ & $\mathbf{4 . 2 3}$ & $\mathbf{4 . 4 5}$ & $\mathbf{6 5} \%$ & $\mathbf{4 . 1 6}$ & $\mathbf{4 . 2 0}$ & $\mathbf{4 . 3 4}$ & $\mathbf{6 3} \%$ \\
\hline
\end{tabular}

Table 3: Human evaluation. Left: Yelp dataset. Right: Yahoo dataset.

\begin{tabular}{ccccc}
\hline Models & Transfer $\uparrow$ & BLEU $\uparrow$ & PPL $\downarrow$ & RPPL $\downarrow$ \\
\hline CAE & $86.9 \%$ & 22.51 & 21.6 & 57.0 \\
\hline w/o cycle-consistency & $88.2 \%$ & 14.5 & 24.4 & 60.6 \\
w/o discriminators & $99.2 \%$ & 0.2 & 25.7 & 701.1 \\
\hline
\end{tabular}

Table 4: Ablation study on Yelp dataset.

The template-based method again achieves the highest BLEU score but it fails to generate meaningful and fluent sentences (very high PPL and RPPL). The reason for this is because simply replacing the style words with unreasonable words may generate senmantically incorrect sentences. For example, the template-based method transfers a source sentence of "is harrison ford married ?" into "is a state in the married ?", which is meaningless. The template-based method also achieves the highest transfer which is different with the results on the Yelp dataset. The reason is that it is easier to differentiate style words from content words in the Yahoo topic dataset than the Yelp dataset which makes it more accurate for the template-based method to substitute style words. It can be observed that the template-based method achieves high BLEU scores at the cost of fluency and semantic correctness. Taking all the four metrics into consideration, we believe that our approach performs better than all the baselines.

\subsubsection{Human evaluation}

Table 3 shows the results of human evaluation. The CAE achieves the highest style transfer, content preservation and fluency score on both datasets. It also obtains the highest comprehensive successful rate of style transfer in terms of Suc.

\subsection{Ablation study}

We further conducted ablation experiments on the Yelp dataset to study the effect of the cycle-consistent constraint and discriminators in CAE. Table 4 shows the results. When we disable the cycle-consistent constraint, we can train the model successfully. However, it leads to significant drop in BLEU and higher PPL and RPPL with marginal improvement in Transfer compared to the full CAE, which again confirms that the cycle-consistent constraint is helpful for content mode preservation. When we disable the discriminators, the model cannot preserve content, obtaining terribly low BLEU and high RPPL, indicating complete mode collapse. Without the discriminators, the CAE has no constraint and guidance to learn the transformation functions, which is prone to mode collapse. Serious mode collapse results in poor content preservation. We notice that most transferred sentences in the "negative" style contain words "not" or "disappointed", while most generated sentences in the "positive" style have the word "good". The discriminators are important for preventing the model from collapse and hence further preserving the content.

\subsection{Analyses}

\subsubsection{Style-transferred sentences}

We display some examples to look into the differences between the CAE and previous approach ARAE in Table 5 . In the first example in Table 5, we can clearly see that CAE correctly detects the sentiment words "great" and "wonderful" and successfully transfers the positive sentiment to the negative sentiment by changing the two words into negative words "horrible" and "awful". It is worth noting that CAE 


\begin{tabular}{|c|c|c|}
\hline Models & Positive $\rightarrow$ Negative & Negative $\rightarrow$ Positive \\
\hline Source & it has a great atmosphere, with wonderful service. & i have n't received any response to anything. \\
\hline ARAE & it has no taste, with a complete jerk. & i have n't received any problems to please \\
\hline CAE & it has a horrible atmosphere, with awful service. & i have been pleased with a wonder time. \\
\hline Source & the steak was really juicy with my side of salsa to balance the flavor. & my corn beef hash was mushy with uncooked veggies. \\
\hline ARAE & the steak was really bland with the sauce and mashed potatoes. & my boyfriend brought shrimp and eggs with mushrooms \\
\hline & & were amazing . \\
\hline CAE & the steak was really dry with my sauce on the salsa. & my beef hash was juicy and tender. \\
\hline Models & Entertainment \& Music $\rightarrow$ Politics \& Government & Politics \& Government $\rightarrow$ Entertainment \& Music \\
\hline Source & how do you publish a song? & who do you think will be next president of the u. s? \\
\hline ARAE & how do you react a song? & who do you think will be next to u ? \\
\hline CAE & how do you handle a war? & who do you think will be next american idol ? \\
\hline Source & $\begin{array}{l}\text { do you know a website that you can find people who want to } \\
\text { join bands? }\end{array}$ & what is the gdp of the us currently? \\
\hline ARAE & do you think that you can find a person who is in prison? & what is the largest of the us currently? \\
\hline CAE & do you know what a website that you can get to join the military? & what is the name of the band currently? \\
\hline
\end{tabular}

Table 5: Style-transferred examples. Upper: from the Yelp dataset. Bottom: from the Yahoo dataset.

preserves the substance of the source sentence during the successful style (sentiment) transfer. In contrast, ARAE fails to keep the background and the major content of original sentences when it struggles to change the style of them not only in the first example but also in other examples.

Examples from Yahoo dataset again demonstrate the advantages of CAE in both style transfer and content preservation over ARAE. It can be obliviously found that CAE is able to learn the patterns of the original questions and to change the topic from "Entertainment \& Music" to "Politics \& Government" or vice versa in the frame of the learned patterns.

\subsubsection{Comparison with the nearest neighbour sequences from training data}

We compared the transferred sequences with the nearest-neighbor sequences from training data based on Jaccard distance (word-level intersection over union for two sequences). The results are listed in Table 6. The transferred sentences are very different from the retrieved nearest sentences in both syntax and semantics. Additionally, they are also very fluent. This suggests that CAE is capable of learning the style knowledge from training instances and generalizing the learned knowledge to generate style-transferred sentences from given source sentences.

\begin{tabular}{l|l}
\hline Transferred sentences & Nearest neighbour in training data \\
\hline it has a horrible atmosphere, with awful service . & horrible atmosphere, horrible service . \\
\hline definitely a waste of time for sushi in las vegas ! & best sushi in las vegas ! \\
\hline the steak was really dry with my sauce on the salsa . & the philly was dry with no sauce . \\
\hline
\end{tabular}

Table 6: Comparison between transferred sentences generated by CAE and nearest-neighbour sentences.

\section{Conclusion}

We have presented a novel approach, CAE, to unsupervised text style transfer from non-parallel text. We learn latent representations for sequences in different styles with LSTM autoencoders. The learned representations are transferred from their original style to another style via adversarial transfer networks. The transfer networks are equipped with a cycle-consistent constraint to guarantee content preservation during style transfer. Experiments and analyses on the Yelp and Yahoo datasets sufficiently demonstrate the powerful style transfer ability of CAE with good fluency and content preservation against previous methods. 


\section{References}

Samuel R. Bowman, Luke Vilnis, Oriol Vinyals, Andrew Dai, Rafal Jozefowicz, and Samy Bengio. 2016. Generating sentences from a continuous space. In Proceedings of The 20th SIGNLL Conference on Computational Natural Language Learning, pages 10-21. Association for Computational Linguistics.

Pierre Colombo, Wojciech Witon, Ashutosh Modi, James Kennedy, and Mubbasir Kapadia. 2019. Affect-driven dialog generation. In Proceedings of the 2019 Conference of the North American Chapter of the Association for Computational Linguistics: Human Language Technologies, Volume 1 (Long and Short Papers), pages 3734 3743, Minneapolis, Minnesota, June. Association for Computational Linguistics.

Ning Dai, Jianze Liang, Xipeng Qiu, and Xuanjing Huang. 2019. Style transformer: Unpaired text style transfer without disentangled latent representation. In Proceedings of the 57th Annual Meeting of the Association for Computational Linguistics, pages 5997-6007, Florence, Italy, July. Association for Computational Linguistics.

Zhenxin Fu, Xiaoye Tan, Nanyun Peng, Dongyan Zhao, and Rui Yan. 2018. Style transfer in text: Exploration and evaluation. In Thirty-Second AAAI Conference on Artificial Intelligence, pages 663-670.

Leon A Gatys, Alexander S Ecker, and Matthias Bethge. 2015. A neural algorithm of artistic style. arXiv preprint arXiv:1508.06576.

Ian Goodfellow, Jean Pouget-Abadie, Mehdi Mirza, Bing Xu, David Warde-Farley, Sherjil Ozair, Aaron Courville, and Yoshua Bengio. 2014. Generative adversarial nets. In Advances in neural information processing systems, pages 2672-2680.

Junxian He, Xinyi Wang, Graham Neubig, and Taylor Berg-Kirkpatrick. 2020. A probabilistic formulation of unsupervised text style transfer. In International Conference on Learning Representations.

R Devon Hjelm, Athul Paul Jacob, Tong Che, Adam Trischler, Kyunghyun Cho, and Yoshua Bengio. 2018. Boundary-seeking generative adversarial networks. In International Conference on Learning Representations.

Sepp Hochreiter and Jürgen Schmidhuber. 1997. Long short-term memory. Neural computation, 9(8):1735-1780.

Zhiting Hu, Zichao Yang, Xiaodan Liang, Ruslan Salakhutdinov, and Eric P Xing. 2017. Toward controlled generation of text. In International Conference on Machine Learning, pages 1587-1596.

Yufang Huang, Joel C. Park, Kelly M. Axsom, Lakshminarayanan Subramanian, and Yiye Zhang. 2020. Dice: Deep significance clustering for outcome-driven stratification. medRxiv.

Armand Joulin, Edouard Grave, Piotr Bojanowski, and Tomas Mikolov. 2017. Bag of tricks for efficient text classification. In Proceedings of the 15th Conference of the European Chapter of the Association for Computational Linguistics: Volume 2, Short Papers, pages 427-431. Association for Computational Linguistics.

Guillaume Lample, Sandeep Subramanian, Eric Smith, Ludovic Denoyer, Y-Lan Boureau, et al. 2019. Multipleattribute text rewriting. In International Conference on Learning Representations.

Juncen Li, Robin Jia, He He, and Percy Liang. 2018. Delete, retrieve, generate: a simple approach to sentiment and style transfer. In Proceedings of the 2018 Conference of the North American Chapter of the Association for Computational Linguistics: Human Language Technologies, Volume 1 (Long Papers), pages 1865-1874. Association for Computational Linguistics.

Fuli Luo, Peng Li, Jie Zhou, Pengcheng Yang, Baobao Chang, Zhifang Sui, and Xu Sun. 2019. A dual reinforcement learning framework for unsupervised text style transfer. In Proceedings of the 28th International Joint Conference on Artificial Intelligence, IJCAI 2019.

A. Mikołajczyk and M. Grochowski. 2018. Data augmentation for improving deep learning in image classification problem. In 2018 International Interdisciplinary PhD Workshop (IIPhDW), pages 117-122, May.

Cicero Nogueira dos Santos, Igor Melnyk, and Inkit Padhi. 2018. Fighting offensive language on social media with unsupervised text style transfer. In Proceedings of the 56th Annual Meeting of the Association for Computational Linguistics (Volume 2: Short Papers), pages 189-194. Association for Computational Linguistics.

Shereen Oraby, Lena Reed, Shubhangi Tandon, Sharath T.S., Stephanie Lukin, and Marilyn Walker. 2018. Controlling personality-based stylistic variation with neural natural language generators. In Proceedings of the 19th Annual SIGdial Meeting on Discourse and Dialogue, pages 180-190. Association for Computational Linguistics. 
Kishore Papineni, Salim Roukos, Todd Ward, and Wei-Jing Zhu. 2002. Bleu: a method for automatic evaluation of machine translation. In Proceedings of 40th Annual Meeting of the Association for Computational Linguistics, pages 311-318. Association for Computational Linguistics, July.

Luis Perez and Jason Wang. 2017. The effectiveness of data augmentation in image classification using deep learning. arXiv preprint arXiv:1712.04621.

Shrimai Prabhumoye, Yulia Tsvetkov, Ruslan Salakhutdinov, and Alan W Black. 2018. Style transfer through back-translation. In Proceedings of the 56th Annual Meeting of the Association for Computational Linguistics (Volume 1: Long Papers), pages 866-876. Association for Computational Linguistics.

Mingyue Shang, Piji Li, Zhenxin Fu, Lidong Bing, Dongyan Zhao, Shuming Shi, and Rui Yan. 2019. Semisupervised text style transfer: Cross projection in latent space. In Proceedings of the 2019 Conference on Empirical Methods in Natural Language Processing and the 9th International Joint Conference on Natural Language Processing (EMNLP-IJCNLP), pages 4939-4948.

Tianxiao Shen, Tao Lei, Regina Barzilay, and Tommi Jaakkola. 2017. Style transfer from non-parallel text by cross-alignment. In Advances in Neural Information Processing Systems, pages 6830-6841.

Liyue Shen, Wentao Zhu, Xiaosong Wang, Lei Xing, John M Pauly, Baris Turkbey, Stephanie Anne Harmon, Thomas Hogue Sanford, Sherif Mehralivand, Peter Choyke, et al. 2020a. Multi-domain image completion for random missing input data. arXiv preprint arXiv:2007.05534.

Tianxiao Shen, Jonas Mueller, Regina Barzilay, and Tommi Jaakkola. 2020b. Educating text autoencoders: Latent representation guidance via denoising. ICML.

Akhilesh Sudhakar, Bhargav Upadhyay, and Arjun Maheswaran. 2019. "transforming" delete, retrieve, generate approach for controlled text style transfer. In Proceedings of the 2019 Conference on Empirical Methods in Natural Language Processing and the 9th International Joint Conference on Natural Language Processing (EMNLP-IJCNLP), pages 3269-3279, Hong Kong, China, November. Association for Computational Linguistics.

Ilya Sutskever, Oriol Vinyals, and Quoc V Le. 2014. Sequence to sequence learning with neural networks. In Advances in neural information processing systems, pages 3104-3112.

Chen Wu, Xuancheng Ren, Fuli Luo, and Xu Sun. 2019. A hierarchical reinforced sequence operation method for unsupervised text style transfer. In Proceedings of the 57th Conference of the Association for Computational Linguistics, ACL 2019, Florence, Italy, July 28-August 2, 2019, Volume 1: Long Papers, pages 4873-4883.

Jingjing Xu, Xu SUN, Qi Zeng, Xiaodong Zhang, Xuancheng Ren, Houfeng Wang, and Wenjie Li. 2018. Unpaired sentiment-to-sentiment translation: A cycled reinforcement learning approach. In Proceedings of the 56th Annual Meeting of the Association for Computational Linguistics (Volume 1: Long Papers), pages 979-988, Melbourne, Australia, July. Association for Computational Linguistics.

Junbo Zhao, Yoon Kim, Kelly Zhang, Alexander Rush, and Yann LeCun. 2018. Adversarially regularized autoencoders. In Jennifer Dy and Andreas Krause, editors, Proceedings of the 35th International Conference on Machine Learning, volume 80 of Proceedings of Machine Learning Research, pages 5902-5911, Stockholmsmässan, Stockholm Sweden, 10-15 Jul. PMLR.

Jun-Yan Zhu, Taesung Park, Phillip Isola, and Alexei A Efros. 2017. Unpaired image-to-image translation using cycle-consistent adversarial networks. In Proceedings of the IEEE international conference on computer vision, pages 2242-2251.

Wentao Zhu, Xiang Xiang, Trac D Tran, Gregory D Hager, and Xiaohui Xie. 2018. Adversarial deep structured nets for mass segmentation from mammograms. In 2018 IEEE 15th International Symposium on Biomedical Imaging (ISBI 2018), pages 847-850. IEEE.

Wentao Zhu, Andriy Myronenko, Ziyue Xu, Wenqi Li, Holger Roth, Yufang Huang, Fausto Milletari, and Daguang $\mathrm{Xu}$. 2020. Neurreg: Neural registration and its application to image segmentation. In The IEEE Winter Conference on Applications of Computer Vision, pages 3617-3626. 